Me. Elizabeth Akemi Nishio, Prof. Dra. Maria Lucia Cardoso, Prof. Dra. Maria D’Innocenzo

\title{
Método para avaliação do modelo de gestão de serviço de enfermagem hospitalar
}

\author{
Eixo: Gestão em Saúde
}

\begin{abstract}
Introdução - Modelos são representações da interação entre conceitos, mostrando padrões (1). Segundo Chinn e Jacobs (2) conceito significa "uma formulação mental complexa de um objeto, propriedade ou acontecimento, originária da percepção e experiências individuais". Os conceitos mais utilizados que determinam a prática de enfermagem são: o homem e o indivíduo, sociedade/e o ambiente, saúde e a enfermagem. O paciente é o centro dos conceitos de enfermagem. Sob estes aspectos existem várias teorias de enfermagem que utilizadas combinadas ou não, determinam às práticas de enfermagem e o modelo de enfermagem ${ }^{(1)}$. Além das teorias de enfermagem relacionadas ao processo assistencial, no processo gerencial, a enfermagem brasileira geralmente utiliza-se de teorias administrativas para planejamento, organização, dimensionamento e gestão do corpo de enfermagem, tais como: a Teoria Geral da Administração, um modelo administrativo racional, no qual o planejamento, organização, direção e controle são para atingir os objetivos da instituição, as estruturas são rígidas e fixas com normas, regras, supervisão e, o modelo histórico-social no qual a gerencia além da organização e controle dos processos de trabalho da instituição hospitalar possui o entendimento para atender as necessidade da saúde da população, traduz as políticas públicas ou privadas (3). Os Serviços de Enfermagem (SE) dos hospitais gerenciados pelo Núcleo Corporativo da Enfermagem da OSS-SPDM, há 20 anos, estabelece um padrão de modelo de gestão de serviços de enfermagem em 15 hospitais, como pesquisadora principal do estudo em desenvolvimento da tese doutorado, propõe-se identificar e avaliar o tipo de modelo de gestão praticado sob a percepção dos enfermeiros que trabalham nos hospitais gerenciados e com os resultados haverá oportunidade para ponderação e adoção de melhorias na gestão dos SE.
\end{abstract}

Objetivo- Avaliar o modelo de gestão do serviço de enfermagem dos hospitais gerenciados pela OSS-SPDM.

Método- Estudo observacional, tipo estudo de caso, quantitativo, transversal e população censitária. Campo de Estudo-15 Serviços de Enfermagem Hospitalar. População do estudo- censitário, enfermeiros contratados e ativos. Coleta de dados: aplicação de dois instrumentos para coleta de dados: caracterização dos enfermeiros e Index of Professional Nursing Governance IPNG, versão Brasileira ${ }^{(4)}$, elaborado por Hess ${ }^{(5)}$,mede como as ações são exercidas no modelo de gestão do serviço hospitalar e a percepção dos enfermeiros sobre a gestão, com 86 itens, nas dimensões: Pessoal, Informação, Recursos, Participação, Prática e Objetivos e Resolução de Conflitos. A coleta será via email, convite para participação da pesquisa, com os objetivos, finalidade e procedimentos do estudo, confidencialidade descritos, Termo de Consentimento Livre e Esclarecido e os instrumentos de coleta para as respostas.

Dados armazenados no software REDcap. Estudo está vinculado ao GEPAV-SE do PG da EPE/ UNIFESP e parte da tese de doutorado do autor principal.

Referências: 1- George JB. Teorias de Enfermagem, tradução: Regina Machado Garces, Artes Médicas, Porto Alegre, 1993. 2- Chinn PL \& Jacobs MK. Theory and Nursing. A Systematic Approach, St. Louis: C.V. Mosby, 1983, p.200. 3- Felli VEA, Peduzzi M. O trabalho gerencial em enfermagem pag.7-8. Coordenadora Kurcgant P. Gerenciamento em Enfermagem, Guanabara Koogan, RJ, 2005.4- Oliveira RJT.Governança da prática profissional de enfermagem: revisão sistemática qualitativa e adaptação do IPNG para uso no Brasil, [dissertação Mestrado Acadêmico em Enfermagem], UFSC, 2016. 5 - Hess RG, Slicing and Dicing shared governance in and around the numbers. Nursing Administration Quartely, 2011 